\title{
Dermatomyositis With or Without Anti-Melanoma Differentiation-Associated Gene 5 Antibodies
}

\section{Common Interferon Signature but Distinct NOS2 Expression}

\author{
Yves Allenbach, ${ }^{* \dagger \ddagger}$ Gaëlle Leroux, ${ }^{\dagger}$ Xavier Suárez-Calvet, ${ }^{\S}$ Corinna Preusse, ${ }^{*}$ Eduard Gallardo, ${ }^{\S}$ Baptiste Hervier, ${ }^{\dagger \ddagger}$ Aude Rigolet, \\ Miguel Hie, ${ }^{\text {" Debora Pehl, }{ }^{*} \text { Nicolas Limal, }}$ Peter Hufnagl, ${ }^{* *}$ Norman Zerbe, ${ }^{* *}$ Alain Meyer, ${ }^{\dagger \dagger}$ Jessie Aouizerate, ${ }^{,+}$

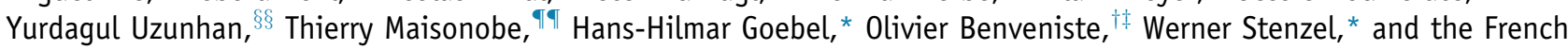 \\ Myositis Network

\begin{abstract}
From the Departments of Neuropathology* and Pathology, ** Charité-Universitätsmedizin, Berlin, Germany; the Departments of Internal Medicine and Clinical Immunology and Inflammation, Immunopathology, and Biotherapy, ${ }^{\dagger}$ and Internal Medicine $2,{ }^{\Uparrow}$ Pitié-Salpêtrière University Hospital, Assistance Publique-Hôpitaux de Paris, Sorbonne Universities, Pierre et Marie Curie University, Paris, France; U974, INSERM, ${ }^{\ddagger}$ Sorbonne Universities, University Pierre et Marie-Curie, Paris, France; the Neuromuscular Diseases Unit, ${ }^{\S}$ Neurology, Hospital de la Santa Creu I Sant Pau, Universita Autonoma de Barcelona and Institute de Recerca Sant Pau, Barcelona, Spain; the Department of Internal Medicine," Assistance Publique-Hôpitaux de Paris, and the Department of Pathology, ${ }^{\ddagger \dagger}$ Mondor Hospital, Creteil, France; the Department of Physiology ${ }^{\dagger \dagger}$ Strasbourg University Hospital, Strasbourg, France; the Department of Pneumology, Avicennes University Hospital, ${ }^{\S \S}$ Assistance Publique-Hôpitaux de Paris, Bobigny, France; and the Department of Neuropathology, "Ti Pitié-Salpêtrière University Hospital, Assistance Publique-Hôpitaux de Paris, Paris, France
\end{abstract}

Accepted for publication November 16, 2015.

Address correspondence to Yves Allenbach, M.D., Ph.D., Service de Médecine Interne 1, Centre de Référence des Maladies Neuromusculaires Paris Est, Groupe Hospitalier Pitié Salpêtrière, Assistance Publique Hôpitaux de Paris, 83 Blvd de l'Hôpital, Paris Cedex 13, France.

E-mail: yves.allenbach@aphp.fr.

\begin{abstract}
The anti-melanoma differentiation-associated gene 5 (MDA5) autoantibody is specifically associated with dermatomyositis (DM). Nevertheless, anti-MDA $5^{+}$-patients experience characteristic symptoms distinct from classic DM, including severe signs of extramuscular involvement; however, the clinical signs of myopathy are mild or even absent. The morphological and immunological features are not yet described in adulthood. Data concerning the pathophysiology of anti-MDA5 DM are sparse; however, the importance of the interferon (IFN) type I pathway involved in DM has been shown. Our aim was to define morphological alterations of the skeletal muscle and the intrinsic immune response of anti-MDA5-positive DM patients. Immunohistological and RT-PCR analysis of muscle biopsy specimens from anti-MDA5 and classic DM were compared. Those with anti-MDA5 DM did not present the classic features of perifascicular fiber atrophy and major histocompatibility complex class I expression. They did not show significant signs of capillary loss; tubuloreticular formations were observed less frequently. Inflammation was focal, clustering around single vessels but significantly less intense. Expression of IFN-stimulated genes was up-regulated in anti-MDA5 DM; however, the IFN score was significantly lower. Characteristic features were observed in anti-MDA5 DM and not in classic DM patients. Only anti-MDA5 DM showed numerous nitric oxide synthase 2-positive muscle fibers with sarcoplasmic colocalization of markers of regeneration and cell stress. Anti-MDA5-positive patients demonstrate a morphological pattern distinct from classic DM. (Am J Pathol 2016, 186: 691-700; http://dx.doi.org/10.1016/j.ajpath.2015.11.010)
\end{abstract}

Dermatomyositis (DM) is a group of idiopathic inflammatory myopathies defined by skin rash, proximal weakness, and specific pathological muscle features. ${ }^{1}$ Characteristically, these encompass the following: perivascular inflammation in the perimysium, perifascicular fiber atrophy, sarcolemmal major histocompatibility complex (MHC) class I staining with a perifascicular enhancement, and microvascular pathology, including capillary enlargement,

Supported by Société Française de Médecine Interne, Association Française contre les Myopathies (A.F.M.).

O.B. and W.S. contributed equally to this work as senior authors

Disclosures: None declared. 
capillary loss, deposition of membrane attack complex (C5b-9) on vascular endothelial cells, and tubuloreticular inclusions in endothelial cells. ${ }^{1,2}$ The pathophysiology of the disease is not fully understood. Hence, on the basis of results of transcriptome analysis of skeletal muscle, it has been emphasized that interferon (IFN) pathways seem to play an important role. ${ }^{3,4}$ Also, a specific vasculopathy associated with molecular mechanisms of hypoxia has been described in detail.

Nevertheless, DM encompasses an inhomogeneous group of disorders because a large spectrum of different extramuscular manifestations can be observed, and the muscle involvement is variable, even absent sometimes: the so-called amyopathic DM. ${ }^{6}$

Different DM-specific autoantibodies have been reported so far, and it has been postulated that their presence is associated with homogeneous clinical subgroups of $\mathrm{DM}^{7}$ Anti-melanoma differentiation-associated gene 5 (MDA5; official symbol IFIH1) is one of them, and the presence of anti-MDA5 antibodies $\left(\mathrm{MDA}^{+}\right.$) delineates a clinical syndrome characterized by mild or absent muscle weakness [with mild or no increase of creatine kinase levels (ie, the prototype of amyopathic DM)]. Extramuscular manifestations are predominant, including severe interstitial lung disease and atypical but characteristic skin lesions with skin ulcers, palmar papules, and/or mechanic's hands. ${ }^{7}$ Thus, MDA $5^{+}$patients harbor a characteristic clinical phenotype, at variance from the classic form of DM, raising the question of the role of anti-MDA5 antibodies, and the involvement of separate underlying molecular mechanisms. It appeared that there are ethnic variations in frequency of anti-MDA5 positivity because most of the cases have been described from Asian populations; however, the associated clinical phenotype in different ethnicities has to be further explored. ${ }^{8}$

To date, muscle pathology of $11 \mathrm{MDA}^{+}$children has been analyzed in comparison to MDA5 ${ }^{-}$juvenile DM patients, ${ }^{9}$ but extensive illustrations have not been reported in adult patients, nor have molecular pathways, including immune parameters, been analyzed.

Herein, we hypothesized that specific morphological features and specific molecular pathways occur in MDA5 $5^{+}$ patients' skeletal muscles, in accordance with the unique phenotype of $\mathrm{MDA}^{+}$patients, which is at variance from classic DM patients. We demonstrate that none of the $\mathrm{MDA}^{+}$patients presented the characteristic morphology of classic DM (perifascicular fiber atrophy, characteristic vasculopathy, or important perivascular inflammation). The expression of IFN-stimulated genes was up-regulated, but at lower levels compared with DM patients. In addition, only $\mathrm{MDA}^{+}$patients showed focal nitric oxide synthase 2 (NOS2) positive fibers. NOS2 expression colocalized with markers of regeneration and markers of cell stress, suggesting a protective mechanism in the skeletal muscle.

\section{Materials and Methods}

\section{Patients and Samples}

Ten muscle biopsy specimens from $\mathrm{MDA}^{+}$patients $(n=9$; one patient had two muscle biopsy specimens) were identified in four French university hospitals involved in this retrospective study. Patients were diagnosed on the basis of the positivity of qualitative commercial dot blot assay, performed according to the manufacturer's recommendations (D-tek, Mons, Belgium). Muscle biopsy specimens $(n=7)$ from DM patients $(n=7)$, diagnosed on the basis of morphological and clinical European Neuromuscular Centre criteria, ${ }^{1}$ were included as controls. The serum of the classic DM controls was tested negative for anti-MDA5 antibodies.

Furthermore, the serum of all patients was also tested for presence of Jo-1, anti-PL-7, anti-PL-12, anti-signal recognition particle, and anti-PM/Scl and anti-Mi-2 autoantibodies using commercial kits (D-tek).

For quantitative real-time PCR, gene expression in DM samples was normalized to results obtained in normal muscle [ie, skeletal muscle biopsy specimens without any light microscopic or ultrastructural abnormalities $(n=7)]$. The following patients' characteristics were documented: epidemiological characteristics, presence of muscle weakness, creatine kinase level, presence of typical skin rash of DM (heliotrope periorbital edema; violaceous papules or macules, scaly if chronic, at metacarpopharyngeal and interpharyngeal joints, and other bony prominences; and erythema of chest, neck, and upper back), ${ }^{1}$ and presence of interstitial lung disease. ${ }^{10}$

\section{Histological and Immunohistochemical Analysis}

All frozen samples were analyzed in the Department of Neuropathology (Charité-Universtitätsmedizin, Berlin, Germany), following standardized procedures. Cryostat sections $(7 \mu \mathrm{m}$ thick) of muscle biopsy specimens were used. The following stains were performed: hematoxylin and eosin, periodic acid-Schiff, acid phosphatase, nonspecific esterase, and Gömöri trichrome. Immunohistochemical analysis included using the following antibodies: anti-MHC class I (mouse, clone W6/32; Dako, Glostrup, Denmark), anti-MHC class II (mouse, clone C3/43; Dako), anti-CD3 (rabbit, polyclonal; Dako), anti-CD4 (rabbit, clone SP35; Zytomed, Berlin, Germany), anti-CD8 (mouse, clone C8/144B; Dako), anti-CD68 (mouse, clone EBM11; Dako), anti-CD79a (rabbit, polyclonal; GeneTex, Irvine, CA), antiCD20 (clone 144B; Dako), anti-C5b-9 complex (mouse, clone aE11; Dako), anti-IFN-stimulated gene 15 (ISG15; rabbit, polyclonal; Abcam, Cambridge, UK), retinoic acid-inducible gene 1 (RIG-I; mouse, 2M6F10; Thermo Scientific, Rockford, IL), anti-neural cell adhesion molecule (clone ERIC-1; AbD Serotec, Kidlington, UK), anti-NOS2 (rabbit, polyclonal; GeneTex), anti-heat shock protein 
(HSP) 70 (mouse, clone 3A3; Abcam), and anti-blood dendritic cell antigen 2 (mouse, clone 10E6.1; Millipore, Schwalbach, Germany). An automated slide staining system (BenchMark XT; Ventana Medical Systems, Tucson, AZ) was used. Utrophin (mouse, clone DRP3/20C5; Leica Microsystems, Wetzlar, Germany) staining was used to visualize endomysial capillaries.

\section{Electron Microscopy}

Ultrastructural analysis was performed after fixation in $2.5 \%$ glutaraldehyde for 48 hours at $4^{\circ} \mathrm{C}$, post fixation in $1 \%$ osmium tetroxide, and embedding of the muscle tissue in araldite. Ultrathin sections were stained with uranyl acetate and lead citrate. A P902 electron microscope (Zeiss, Oberkochem, Germany) was used to analyze the specimens.

\section{Histological Quantification}

All slides were retrospectively analyzed blinded to the antibody status by three myopathologists (W.S., Y.A., H.-H.G.). The presence or absence of each item is mentioned in the study. For quantitative analysis, slides were digitized using Pannoramic 250 (3DHistech, Budapest, Hungary) at a resolution of $0.244 \mu \mathrm{m}$ per pixel. Whole slide images were processed using a virtual microscope on the basis of ImageJ software version 6.1.1 (NIH, Bethesda, MD; http://imagej. nih.gov/ij). ${ }^{11}$ The density of immune cells was calculated after counting positive cells from the whole area of the biopsy specimen. For capillary analysis, a sufficient number of randomly selected detail images at $\times 100$ magnification allowed coverage of $50 \%$ to $60 \%$ of the whole cross section of the biopsy. For each sample, the number of capillaries and the minimum Feret diameters were measured automatically. The number of entire cross sections of muscle fibers was counted manually. These data permitted us to calculate the capillary/fiber ratio and capillary density. ${ }^{12}$

\section{Real-Time Quantitative PCR}

For RNA extraction from the muscle tissue samples, RTPCR and quantitative PCR were used with the same methods as described before. ${ }^{13}$ Briefly, the expression levels of gene transcripts were analyzed by quantitative real-time RT-PCR using the $5^{\prime}$-nuclease technology on an ABI PRISM 7900HT Sequence Detection System, and the human TaqMan predeveloped assay reagents (both from Applied Biosystems, Darmstadt, Germany). The assay identification probes are as follows: $2^{\prime}-5^{\prime}$-oligoadenylate synthetase 1 (OAS1), Hs00973637_m1; OAS3, Hs00196324_m1; MDA5, Hs01070332_m1; myxovirus (influenza virus) resistance 1 (MXI), Hs00895608_m1; RIG-I, Hs00204833_m1; IFN- $\gamma$ (IFNG), Hs00989291_m1; and STAT1, Hs01013989_m1. The relative abundance of target transcripts was normalized to the expression levels of the endogenous control gene: phosphoglycerate kinase 1 (PGK1), Hs99999906_m1. For each probe, individual (MDA5 $^{+}$and classic DM patients) data were expressed as relative quantification value. The relative quantification value is equal to $2^{-\Delta \Delta \mathrm{Ct}}$ (ie, normalized fold-change relative to the mean expression for the seven healthy controls combined). As previously described, the median fold-change in expression of six IFN-stimulated genes (ISGs) was given for each patient's biopsy specimen, defining an IFN score. ${ }^{14}$

\section{Immunofluorescence Analysis}

Immunofluorescence analysis was performed as previously described. ${ }^{13}$ Briefly, sections were blocked with the appropriate serum and incubated with the previously mentioned primary antibodies. The secondary antibody was added for 1 hour. The same protocol was repeated with a second primary antibody and an appropriate secondary antibody for double immunofluorescence staining. Images were taken with the Zeiss Observer.Z1 microscope (immunofluorescence), Axiovision software version 4 (Zeiss), or the Olympus (Hamburg, Germany) BX50 microscope, the digital camera DP25, and cell D software version 5.1.

Agreement from the French Ministry of Research (AC 2008-87) was obtained for the collection, analysis, storage, and reuse. All samples were taken for diagnostic purposes. We conducted the research after consideration and approval of the research ethics committee of the Pitié-Salpêtrière Hospital (Paris, France).

\section{Statistical Analysis}

Categorical variables are reported as numbers and/or percentages and were compared using a Fisher's exact test. Quantitative variables are reported as means \pm SD and compared using the Mann-Whitney test. $P<0.05$ was considered significant. Kruskal-Wallis one-way analysis of variance was applied to analyze quantitative differences of mRNA transcripts, using Bonferroni correction of the post hoc tests. GraphPad Prism software version 6.00 (GraphPad Software, La Jolla, CA) was used for analysis.

Table 1 Patient Characteristics

\begin{tabular}{lccl}
\hline Criterion & MDA5 $^{+}$ & Classic DM & $P$ values \\
\hline Age, years* & $44 \pm 17.7$ & $47 \pm 12.1$ & 0.6 \\
Female, \% & 77 & 85 & 1 \\
Muscle weakness & 28 & 100 & 0.003 \\
CK level, IU/L* & $517 \pm 856$ & $2693 \pm 3446$ & 0.03 \\
Interstitial lung disease, $\%$ & 67 & 0 & 0.01 \\
Atypical skin lesions, \% & 90 & 0 & 0.001 \\
\hline
\end{tabular}

The atypical skin lesions correspond to presence of palmar papules, mechanic's hands, and/or skin ulcers.

*Data presented as means \pm SD.

CK, creatine kinase; DM, dermatomyositis; MDA, melanoma differentiation-associated protein. 

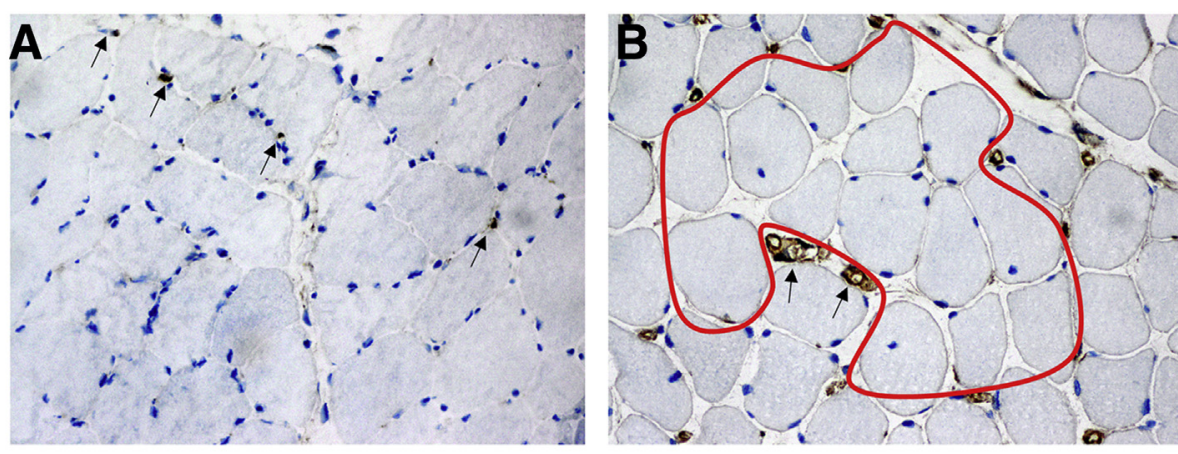

C
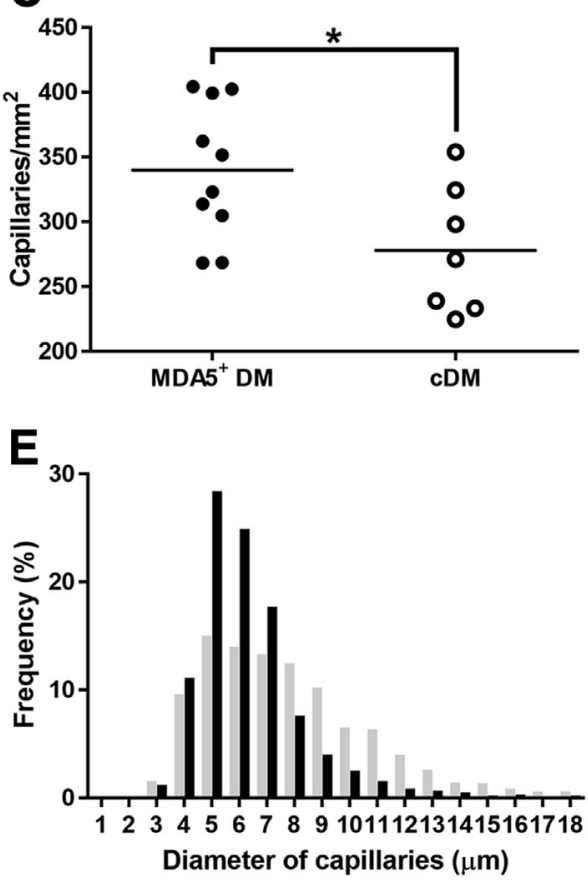

D
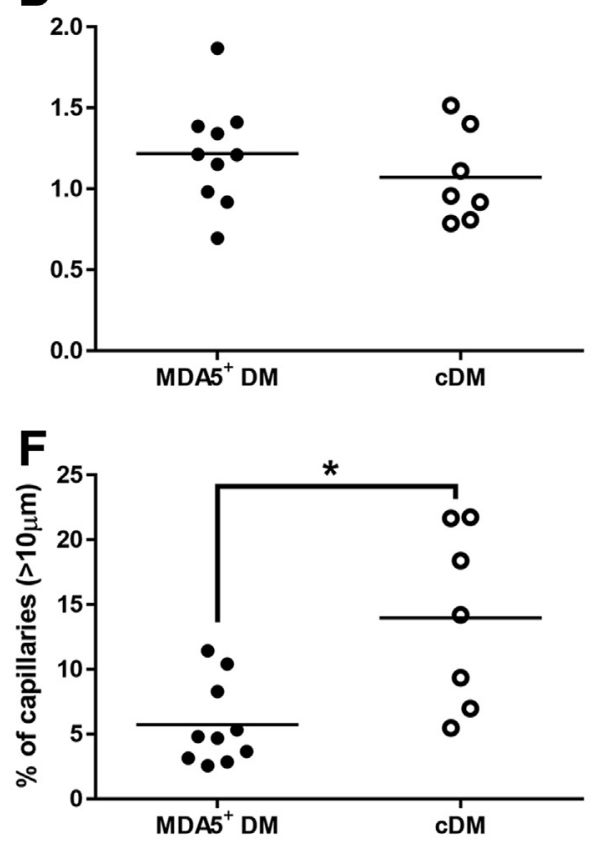

Figure 1 Vasculopathy in $\mathrm{MDA}^{+}$patients. A: Images of muscle fibers from a $\mathrm{MDA5}^{+}$patient showing mild membrane attack complex deposition on few capillaries (arrows); C5b-9 was used. B: Rare capillary loss in a $\mathrm{MDA5}^{+}$patient, delineated by the red outline. Presence of few enlarged capillaries (arrows); utrophin was used to stain the capillaries. C: Significantly lower capillary density in CDM patients. D: No significant difference in capillary/fiber ratios in $\mathrm{MDA5}^{+}$and $\mathrm{CDM}$ patients. E: Quantitative distribution of capillaries depending on their diameter in one representative $\mathrm{MDA}^{+}$patient (black bars) and one representative CDM patient (gray bars). For each case, $>1000$ capillaries were counted. F: Dot plot representing the percentages of enlarged capillaries. G: Ultrastructural evidence of tubuloreticular inclusion in an endothelial cell of a MDA $5^{+}$DM patient. * $P<0.05$. Original magnifications: $\times 400(\mathbf{A}$ and $\mathbf{B}) ; \times 12,000(\mathbf{G})$. CDM, classic dermatomyositis; MDA5, melanoma differentiation-associated gene 5 .

G

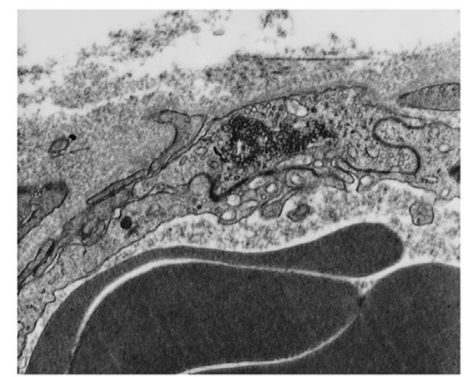

\section{Results}

\section{Characteristics of $\mathrm{MDA}^{+}$and Classic DM Patients}

Patients' characteristics are summarized in Table 1. Patients were mainly female and were aged 40 to 49 years when the muscle biopsy specimen was obtained. Age and sex ratio were not different in $\mathrm{MDA}^{+}$versus classic DM groups. The extramuscular phenotype was different because most $\mathrm{MDA}^{+}$patients experienced interstitial lung disease, whereas interstitial lung disease was absent in controls. In addition, signs of myopathy were more frequent and/or more intense in classic DM patients. Only one quarter of MDA5 ${ }^{+}$ patients experienced verifiable muscle weakness, and only $55 \%$ of them had increased creatine kinase levels. All DM patients presented typical skin manifestations, whereas most MDA5 $^{+}$DM patients experienced characteristic skin lesions uncommon in classic DM (none of the classic DM patients presented atypical skin lesions, such as palmar papules, mechanic's hands, and/or skin ulcers) (Table 1). None of the DM patients harbored anti-synthetase syndrome, two classic DM patients were anti-Mi-2 antibody positive, and none of
$\mathrm{MDA5}^{+}$patients had myositis-specific antibodies other than anti-MDA5.

\section{Vasculopathy in $\mathrm{MDA}^{+}$Patients}

One of the main characteristics of DM pathology is the presence of a characteristic vasculopathy. To assess signs of vasculopathy, we first tested the presence of C5b-9 deposition on endomysial capillaries (Figure 1A). Characteristic C5b-9 deposition was regularly (85\%) detected in classic DM, but this was not the case for MDA5 ${ }^{+}$patients $(30 \%, P=0.049)$.

Capillary rarefaction is a further feature of vasculopathy in DM. Indeed, capillary density was low in classic DM patients $\left(277.9 \pm 49.5\right.$ per $\left.\mathrm{mm}^{2}\right)$, and it was significantly higher $\left(340 \pm 52.3\right.$ per $\left.\mathrm{mm}^{2}, P=0.05\right)$ in $\mathrm{MDA}^{+}$patients (Figure 1, B and C). Focal capillary rarefaction was only rarely noted in $\mathrm{MDA}^{+}$patients (Figure 1B). The capillary/ fiber ratio was slightly elevated in $\mathrm{MDA}^{+}$patients $(1.2 \pm 0.3)$ compared with classic DM $(1.1 \pm 0.2)$; however, it did not reach statistical significance $(P=0.36)$ (Figure 1D). Enlarged capillaries, another sign of 
vasculopathy, were commonly observed in classic DM patients; however, they could only occasionally be observed in $\mathrm{MDA}^{+}$patients (Figure 1B). Indeed, the mean percentage of enlarged capillaries $(>10 \mu \mathrm{m}$ diameter $)$ was high in classic DM patients $(13.9 \% \pm 6.8 \%)$ and was only $5.7 \% \pm 3.2 \%$ in $\mathrm{MDA}^{+}$patients $(P=0.006)$ (Figure $1, \mathrm{E}$ and F). In addition, all classic DM patients had tubuloreticular inclusions within vascular endothelial cells (Figure $1 \mathrm{G}$ ), whereas they were detected in barely $50 \%$ of $\mathrm{MDA5}^{+}$patients $(P=0.04)$. Together, these results show that typical vasculopathy, as found in DM, is not a characteristic feature of $\mathrm{MDA}^{+}$patients.

\section{Skeletal Muscular Fiber Abnormalities in $\mathrm{MDA}^{+}$ Patients}

The presence of perifascicular fiber atrophy is one of the most characteristic signs of DM. This pattern was not observed in $\mathrm{MDA5}^{+}$patient biopsy specimens $(P=0.003)$ (Figure $\left.2 \mathrm{~A}\right)$, except in one case in which a focal mild perifascicular atrophy was noted next to a perivascular infiltrate (data not shown). In contrast, all but one classic DM patient showed the typical perifascicular atrophy $(P=0.003)$ (Figure 2B). In the same line, in $\mathrm{MDA}^{+}$patients, MHC-I expression was essentially focal or absent (Figure 2C), only one patient showed a mild diffuse expression. On the contrary, a diffuse MHC class I expression with reinforcement in perifascicular regions, which is a characteristic feature of DM, was regularly met in classic DM $(P=0.004)$ (Figure $2 \mathrm{D})$.

\section{Inflammation in $\mathrm{MDA}^{+}$Patients}

By definition, DM is a myopathy with inflammatory infiltrates mainly occurring in the perivascular and perimysial areas. All but one $\mathrm{MDA}^{+}$patient harbored inflammatory infiltrates; however, the topographic distribution of these infiltrates differed considerably from classic DM patients. In $\mathrm{MDA}^{+}$patients, infiltrates were clustering focally in the perimysium near a single vessel (Figure 3A), whereas in classic DM, as expected, inflammation was detected multifocally, leading to a more homogeneous inflammatory aspect (Figure 3B). Macrophages were the predominant immune cells in $\mathrm{MDA}^{+}$patients and essentially accumulated only focally in the perimysium as well (Figure 3, C and $\mathrm{D})$. Along that line, cellular density of $\mathrm{T}$ cells $\left(\mathrm{CD}^{+}\right.$ cells) was significantly elevated in classic DM $(35.8 \pm 30$ cells per $\mathrm{mm}^{2}$ ), whereas $\mathrm{T}$ cells were rare in $\mathrm{MDA}^{+}$patients $\left(3.6 \pm 3.1\right.$ cells per $\left.\mathrm{mm}^{2} ; P<0.0001\right)$ (Figure 3E). Among $\mathrm{T}$ cells, $\mathrm{CD} 8^{+} \mathrm{T}$ cells represented $7.7 \pm 7.1$ cells per $\mathrm{mm}^{2}$ in classic DM patients, and were significantly lower in $\mathrm{MDA}^{+}$patients $\left(1.3 \pm 2.5\right.$ cells per $\mathrm{mm}^{2}$; $P<0.01)$. CD4 ${ }^{+}$T cells were detectable more frequently in classic DM $\left(22.4 \pm 5.7\right.$ cells per $\mathrm{mm}^{2}$ versus $2.9 \pm 2.9$ cells per $\mathrm{mm}^{2} ; P<0.001$ ) (Figure 3, F and G). CD20 ${ }^{+}$B-cell density was elevated in classic DM $(8.2 \pm 6.3$ cells per $\mathrm{mm}^{2}$ ), but it was significantly lower in $\mathrm{MDA}^{+}$patients $\left(2.1 \pm 2.8\right.$ cells per $\left.\mathrm{mm}^{2} ; P<0.02\right)$ (Figure $3 \mathrm{H}$ ). Few plasma cells were present in $\mathrm{MDA}^{+}$and classic DM patients, with no significant difference $(0.3 \pm 0.6$ and $1.0 \pm 1.2$ cells per $\mathrm{mm}^{2} ; P=0.1$; data not shown).

\section{IFN Signature in Skeletal Muscle of $\mathrm{MDA5}^{+}$Patients}

We next compared immune pathways in $\mathrm{MDA}^{+}$and classic DM patients and first tested the IFN pathway. We analyzed the expression of six ISGs: $O A S 1$, ISG15, OAS3, $M X I, R I G-I$, and IFIHI. All these genes were considerably up-regulated in both groups compared with normal
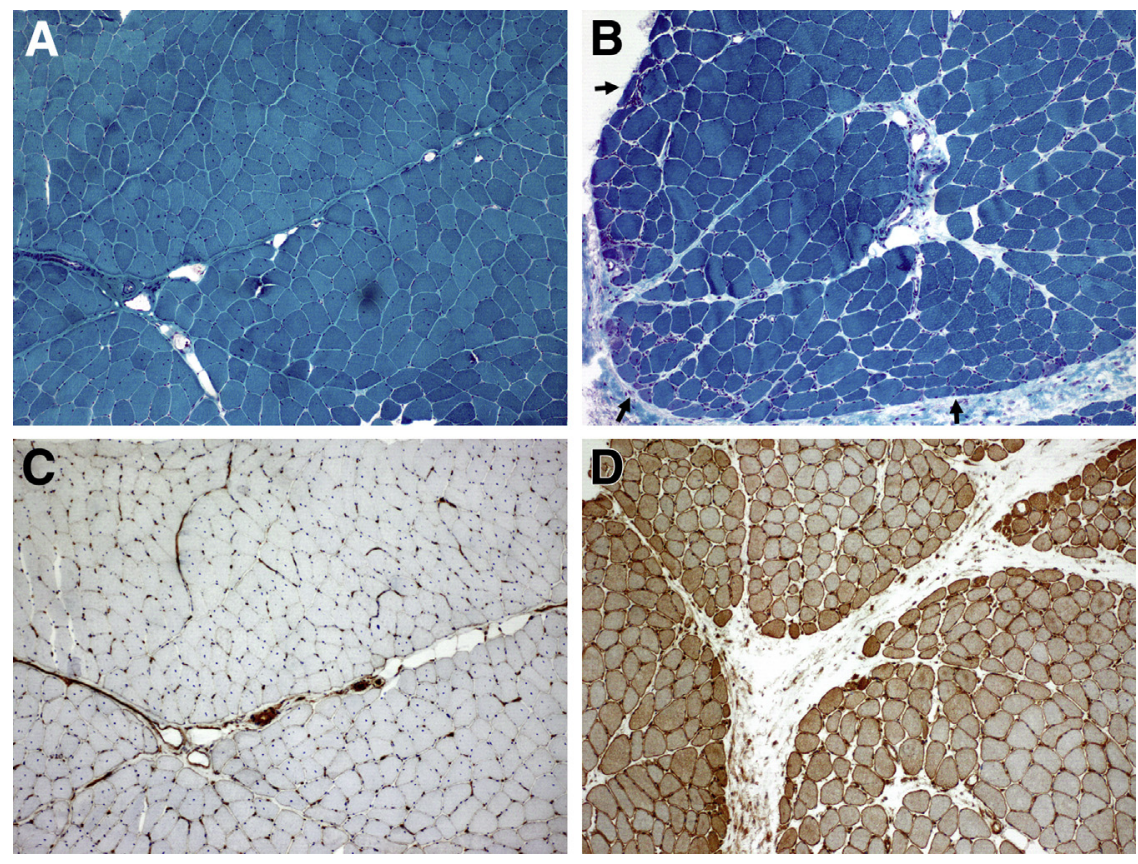

Figure 2 Characteristics of inflammatory myopathy in $\mathrm{MDA}^{+}$patients. A: No significant muscle atrophy in a $\mathrm{MDA}^{+}$patient. B: Myofiber atrophy in the perifascicular region (arrows) in a classic DM patient. A and B: Gömöri trichrome staining was used. C: No sarcolemmal MHC- class I positive immunoreactivity on muscle fibers in a $\mathrm{MDA}^{+}$patient; physiological staining was on endothelial cells. D: Diffuse and strong MHC class I positive immunoreactivity on muscle fibers with a perifascicular reinforcement in a classic DM patient. Original magnification, $\times 100$ (A-D). DM, dermatomyositis; MDA5, melanoma differentiation-associated gene 5; MHC, major histocompatibility complex. 

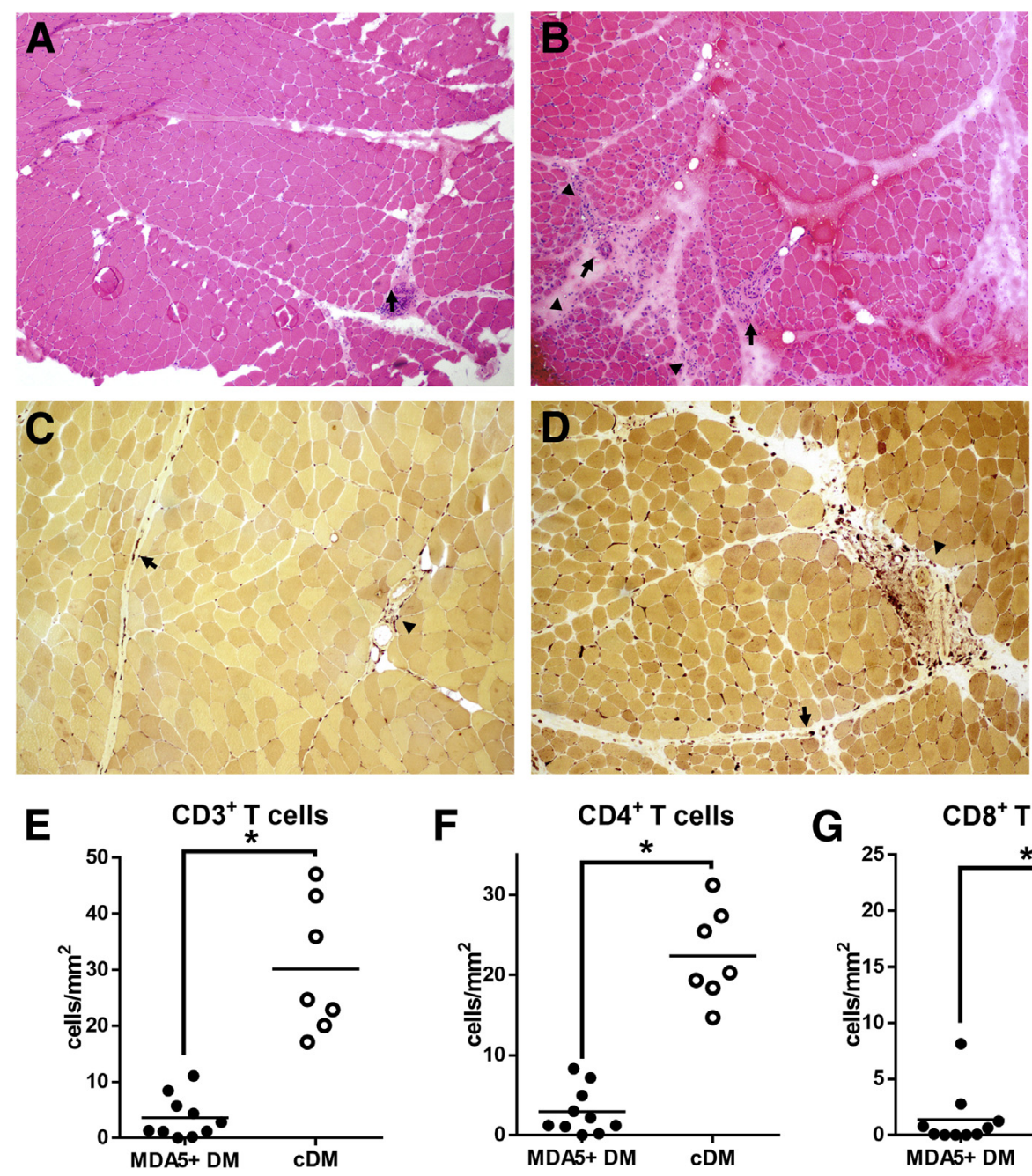

$\mathbf{F}$

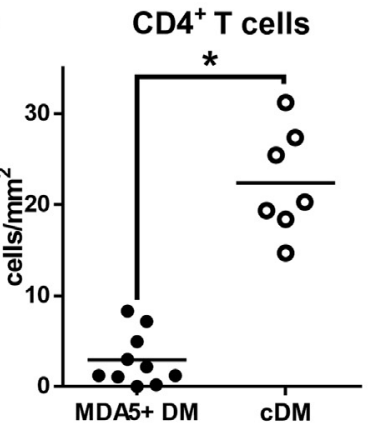

G

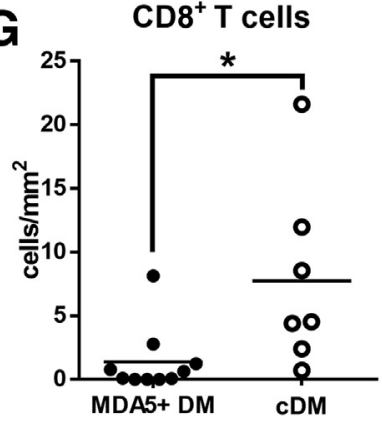

Figure 3 Inflammation in skeletal muscle of $\mathrm{MDA} 5^{+}$patients. A and B: Focal unique inflammatory infiltrate (arrow) clustering around a vessel in the biopsy specimen of skeletal muscle from a $M D A 5^{+}$patient (A). Multifocal inflammatory infiltrates (arrows) in perivascular regions, associated with perimysial inflammation with extension to the endomysium (arrowheads) in a CDM patient (B). Hematoxylin and eosin staining was used. C: Mild inflammatory infiltrate in a $\mathrm{MDA}^{+}$patient composed of macrophages (nonspecific esterase positive cells in dark brown) around a vessel (arrowhead) or in a perifascicular region (arrow). D: Significant amount of macrophages in perifascicular (arrows) and perivascular (arrowheads) areas in a CDM patient. E-H: Significantly lower T-cell density (E), encompassing $\mathrm{CD}^{+}(\mathbf{F})$ and $\mathrm{CD}^{+}(\mathbf{G})$ T-cell subpopulations and $\mathrm{CD}_{20} \mathrm{O}^{+} \mathrm{B}$ cells $(\mathrm{H})$ as well in $\mathrm{MDA}^{+} \mathrm{DM}$ patients compared with $\mathrm{CDM}$ patients. ${ }^{*} P<0.05$. Original magnifications: $\times 80$ (A and $\mathbf{B}) ; \times 100$ (C and D). CDM classic dermatomyositis; DM dermatomyositis; MDA5, melanoma differentiationassociated gene 5 . muscle with high relative quantification values (Figure 4A). Nevertheless, the up-regulation of ISGs was less important in $\mathrm{MDA}^{+}$patients than in classic DM, and the IFN score was significantly lower in $\mathrm{MDA}^{+}$patients $(165.8 \pm 138.2$ versus $590.2 \pm 141.5 ; P<0.004)$ (Figure 4B).

To identify cells involved in the IFN pathway, we first searched for the presence of plasmacytoid dendritic cells (pDCs; stained by anti-blood dendritic cell antigen 2), which are known to secrete a large amount of IFN- $\alpha$. Only occasional pDCs were observed in a minority of MDA5 $5^{+}$and classic DM patients (Supplemental Figure S1). On the other hand, we observed that muscle fibers expressed proteins of the ISG group at different levels. RIG-I and ISG15 were regularly detectable in classic DM, on skeletal muscle fibers in the perifascicular region (Figure 4, C and E). On the contrary, only $50 \%$ of $\mathrm{MDA}^{+}$patients showed a variable degree of RIG-I staining (Figure 4D), and none of the $\mathrm{MDA}^{+}$patients exhibited detectable ISG15 immunoreactivity in muscle fibers (Figure 4F); however, some immune cells showed a positive immunostaining (Figure 4F). Together, these results show that the IFN pathway was also activated to a lesser degree in $\mathrm{MDA}^{+}$patients compared with classic DM patients.

\section{Role of NOS2 Expression and Th1 Immunity in MDA5 ${ }^{+}$ Patients}

The IFN-associated cytokines are considered strong inducers of STAT1, which is required for type 1 helper T-cell (Th1) activation as part of the adaptive immune response. ${ }^{15}$ We tested STAT1 gene expression and found increased levels of STAT1 transcription in $\mathrm{MDA5}^{+}$specimens but less important compared with classic DM patient biopsy specimens (Figure 5A). These results are consistent with the increase of gene expression of the Th1 cytokine IFNG that we observed in both groups, which again was less pronounced in specimens from $\mathrm{MDA}^{+}$patients (Figure 5B). To further investigate Th1 immunity, we also tested NOS2 expression characterizing macrophage activation in a Th1 environment. ${ }^{16}$ We detected numerous $\mathrm{NOS}^{+}$mononuclear infiltrates in both groups, but they were more abundant in the classic DM patients (Figure 5, $\mathrm{B}$ and C). Strikingly, however, specimens from $\mathrm{MDA}^{+}$patients showed a strong $\mathrm{NOS}^{+}$immune reaction on the 
A
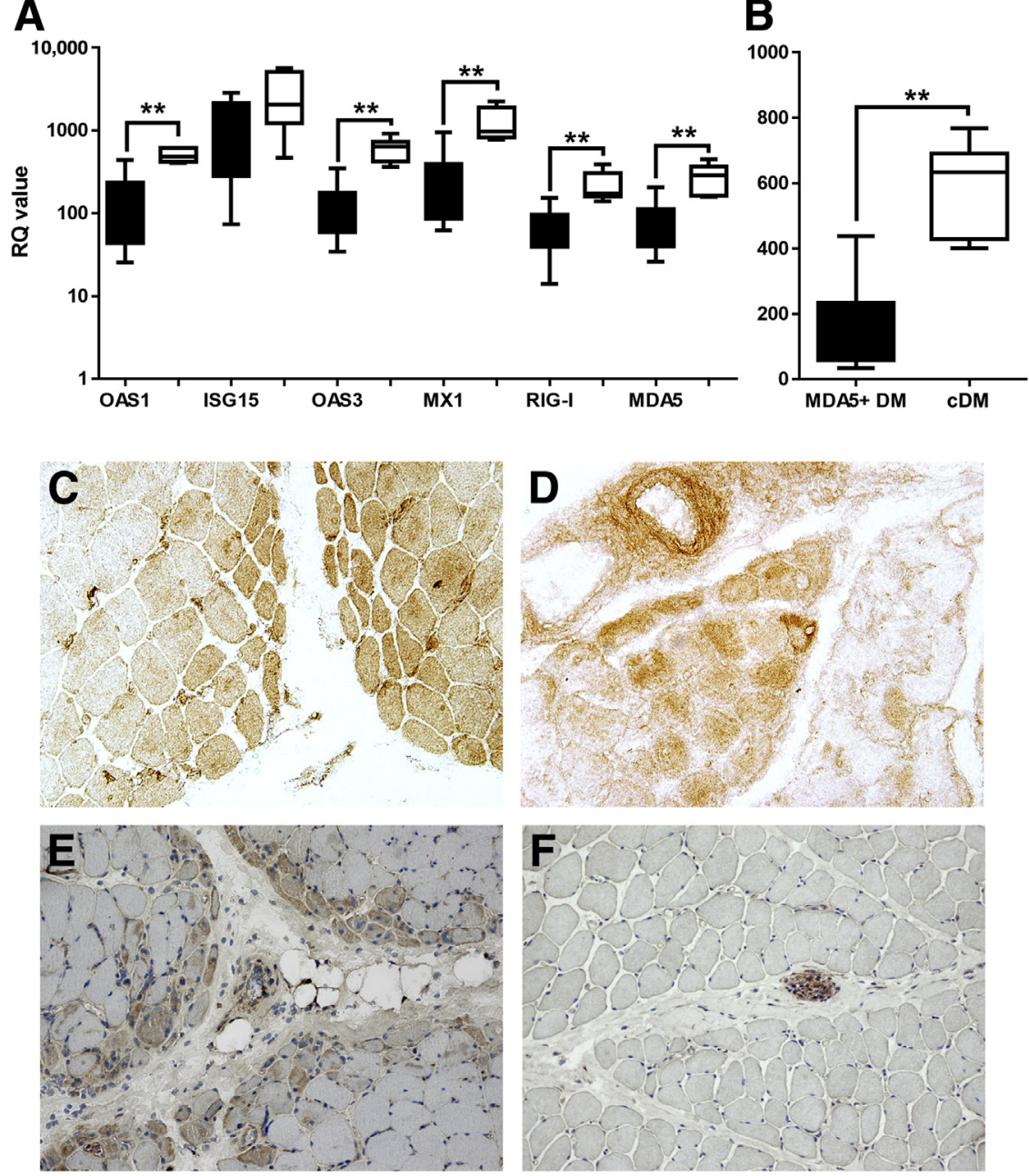

Figure 4 IFN signature in $\mathrm{MDA}^{+} \mathrm{DM}$ patients. A: Box-and-whiskers graph showing RQ values for a panel of six ISGs [2'-5'-oligoadenylate synthetase (OAS)1, ISG15, OAS3, myxovirus (influenza virus) resistance $(M X) 1$, retinoic acid-inducible gene (RIG)1, and IFIH1] measured in skeletal muscle tissue from $\mathrm{MDA}^{+}$(black boxes) and $\mathrm{CDM}$ (white boxes) patients. B: IFN score calculated from the median fold-change of the $R Q$ value for the panel of six ISGs in $\mathrm{MDA}^{+}$(black boxes) and CDM (white boxes) patients. C and D: RIG-I-positive fibers were detected in both $\mathrm{CDM}$ and $\mathrm{MDA} 5^{+} \mathrm{DM}$ biopsy specimens. $\mathbf{E}$ and $\mathbf{F}$ : ISG15-positive myofibers were detectable in the perifascicular region on atrophic fibers in CDM biopsy specimens (E), whereas ISG15 was not detectable in $\mathrm{MDA}^{+}$DM biopsy specimens, but only on inflammatory infiltrates (F). ${ }^{* *} P \leq 0.01$. CDM, classic dermatomyositis; DM, dermatomyositis; IFN, interferon; ISG, IFN-stimulated genes; MDA5, melanoma differentiationassociated gene $5 ; R Q$, relative quantification. sarcoplasm of myofibers; conversely, classic DM patients did not $(P<0.003)$ (Figure 5B). In $\mathrm{MDA}^{+}$patients, numerous $\mathrm{NOS}^{+}$fibers were detectable in fascicles adjacent to perimysial vessels with inflammatory infiltrates (Figure 5D).

Because NOS2 positivity was a striking feature in MDA5 patients' biopsy specimens only, we hypothesized a protective, and not a detrimental, effect of NOS2 in the skeletal muscle. Therefore, we tested for the presence of chaperone proteins associated with protection. Indeed, $\mathrm{NOS}^{+}$fibers in MDA5 ${ }^{+}$ patient biopsy specimens strongly colocalized with HSP70 (Figure 5E). In addition, colocalization with neural cell adhesion molecule was also observed (Figure 5F); as mentioned above, classic DM (MDA5 $\left.{ }^{-}\right)$patients did not show NOS2positive fibers (no colocalization, data not shown). In classic DM, however, only single fibers were NOS2 positive, whereas fibers in the perifascicular region were HSP70 positive and no colocalization was detectable (Figure 5G).

\section{Discussion}

In this study, addressing morphological and immunological aspects in $\mathrm{MDA}^{+}$patients, we show that muscle pathology and the local immune response differ from classic DM patients. The main finding of this study is that we observed a characteristic specific NOS2 expression on the sarcoplasm of HSP70-positive muscle fibers in $\mathrm{MDA}^{+} \mathrm{DM}$ patients, which was absent in classic DM patients. Conversely, the IFN pathway was activated in both groups. We analyzed these observations in the light of the intriguing fact that MDA5 ${ }^{+}$patients show mild signs of myositis but a severe systemic disease.

To our knowledge, there is no comprehensive report documenting muscle pathology in adult $\mathrm{MDA} 5^{+}$patients. Indeed, the MDA5 antibody was the first one specifically associated with clinically amyopathic DM. ${ }^{17}$ Interestingly, Tansley et $\mathrm{al}^{9}$ have also described a reduced severity score in juvenile $\mathrm{MDA}^{+}$patients compared with other MDA5 juvenile DM biopsy specimens.

There are only few reports documenting morphological findings of skeletal muscle biopsy specimens in clinically amyopathic DM patients. ${ }^{5,18,19}$ They postulate that clinically amyopathic DM may serve as a paradigm of vasculopathy, because clinically amyopathic DM muscle biopsy specimens show severe capillary loss. ${ }^{5,19}$ However, in our study, 
A
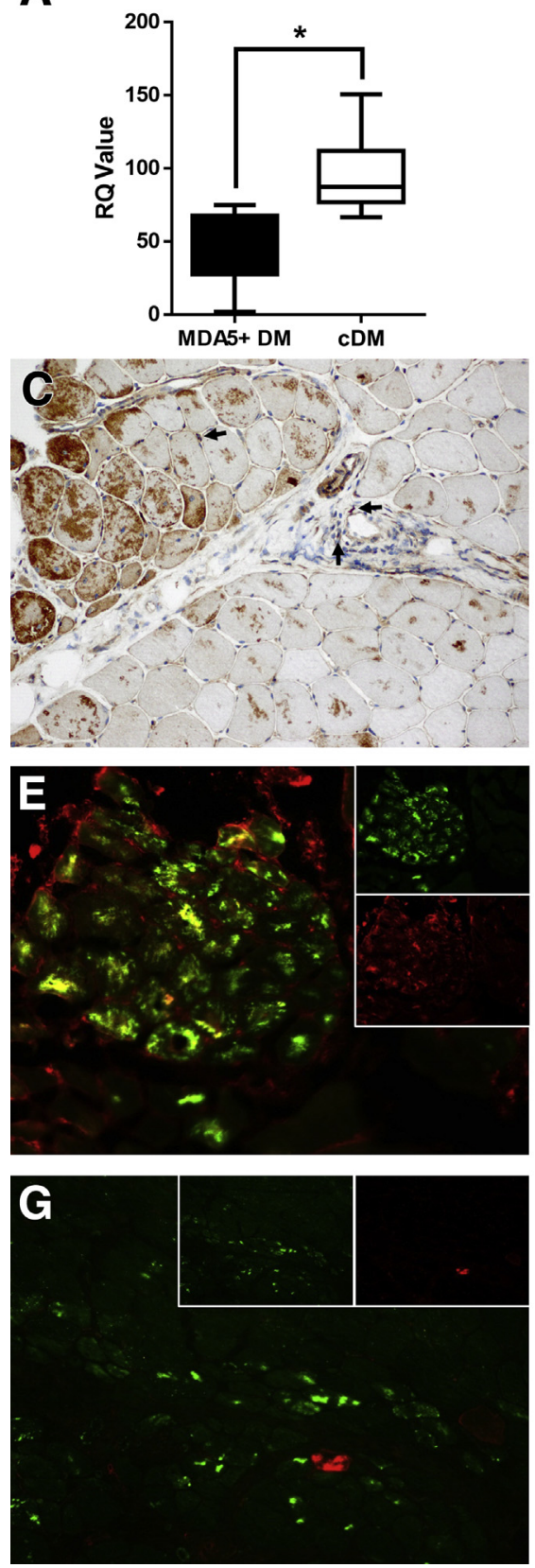

B
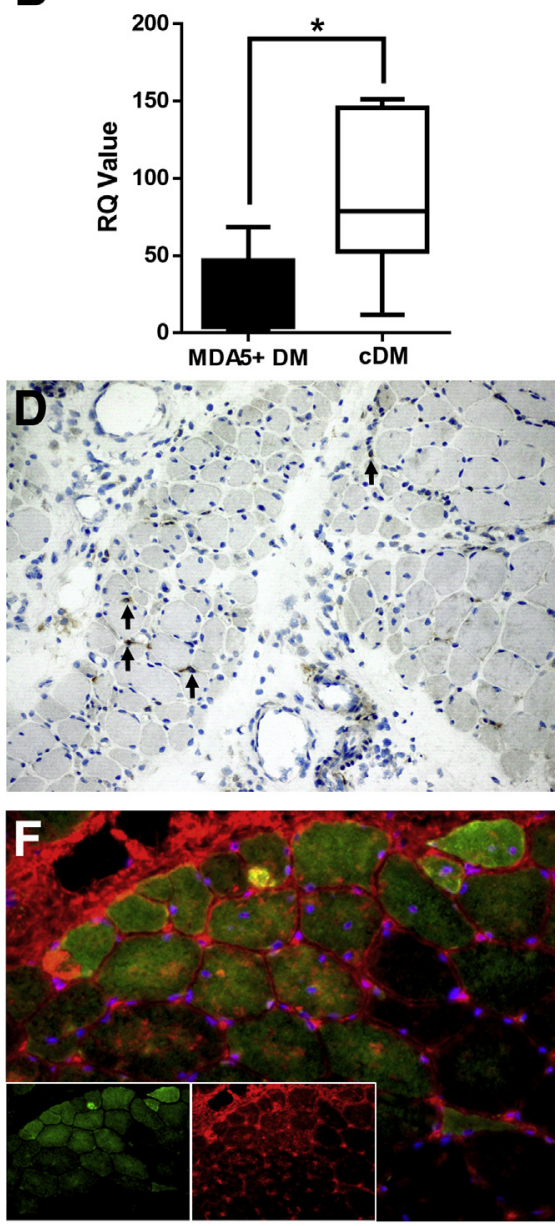

Figure 5 Type 1 helper T-cell immunity and expression of NOS2 in muscle fibers of MDA5 ${ }^{+}$ patients. Box-and-whiskers graph showing $R Q$ values for gene expression of STAT1 (A) and IFNG (B) genes measured in muscle tissue from $M D A 5^{+}$ (black boxes) and CDM patients (white boxes). C: NOS2-positive immune cells corresponding to activated M1 macrophages (arrows), and strong immunoreactivity with NOS2 on numerous muscle fibers in a $\mathrm{MDA}^{+} \mathrm{DM}$ patient. Positive fibers were close to a vessel, and some were surrounded by inflammatory infiltrates. D: NOS2-positive immune cells (arrows) in a cDM patient. No immunostaining on sarcolemma or sarcoplasm of fibers. E: Colocalization of NOS2 (red) positive myofibers with heat shock protein 70 (green) positive myofibers in an $\mathrm{MDA}^{+}$patient illustrated by immunofluorescence. F: Colocalization of NOS2 (green) positive myofibers with neural cell adhesion molecule (red) positive myofibers in an $\mathrm{MDA}^{+}$patient illustrated by immunofluorescence. G: One single fiber shows NOS2 positivity (red) and does not colocalize with perimysially localized atrophic fibers in a CDM patient biopsy specimen. ${ }^{*} P<0.05$. Original magnification, $\times 200 \quad(\mathbf{C}-\mathbf{G}) . \quad$ CDM, classic dermatomyositis; MDA5, melanoma differentiation-associated gene 5; NOS, nitric oxide synthase; RQ, relative quantification.

vasculopathy was less pronounced or absent in comparison to biopsy specimens from classic DM patients without MDA5 antibodies. ${ }^{20}$ Electron microscopy, which has never been reported, revealed that half of the specimens showed tubuloreticular inclusions in endothelial cells, whereas all DM patients showed this feature, as previously reported. ${ }^{2}$

In contrast to classic DM, inflammatory infiltrates were essentially detected focally, clustering around single vessels. This mild quantitative presence of inflammatory cells fits to the mild muscle phenotype in general, including low CK levels. All patients showed immune inflammatory abnormalities, even if clinical signs of myopathy were completely absent.

The IFN signature was highlighted to be of paramount importance in the pathophysiology of $\mathrm{DM} .^{3}$ On the basis of recent publications confirming these results, ${ }^{3,4,21}$ six ISGs were selected and found up-regulated; however, this occurred more strongly in classic DM than in MDA5 ${ }^{+}$ patients. The IFN pathways can be activated by IFN- $\alpha$, a cytokine mainly produced by pDCs; however, we were not able to identify a significant amount of pDCs in both groups, in accordance with what was described by Eloranta et al. ${ }^{22}$ However, others reported significant amounts of pDCs in classic DM. ${ }^{3}$ Consistent with previous studies showing expression of IFN-dependent genes (ISG15, $M X 1$, or $R I G I$ ) at the molecular level in muscle fibers of DM patients, ${ }^{3,4,21}$ we were able to illustrate expression of RIG-I and ISG15 on muscle fibers by immunohistochemistry in $\mathrm{MDA}^{+}$patients. This illustrates that IFN 
pathways also involve muscle fibers per se in addition to immune cells.

The common IFN signature we observed in both groups is not a conflicting result regarding the differences in extramuscular phenotypes and the muscle pathology in DM and $\mathrm{MDA}^{+}$patients. Indeed, differences in the quality or quantity of the IFN response, and/or the existence of specific protective mechanisms in the skeletal muscle, may occur. As an example, type I interferonopathies because of genetically manifest gain of function lead to different clinical phenotypes, depending on their specific mutation, whereas the IFN signature is common. ${ }^{23,24}$

Along that line, expression of NOS2 in muscle fibers of MDA5 $^{+}$DM patients was widespread and strong, largely confined to regenerating fibers in contrast to classic DM patients. Nitric oxide produced by NOS2 comprises two opposing effects of the immune system (namely, regulatory and effector functions). ${ }^{25}$ The significant NOS2 expression we observed in $\mathrm{MDA}^{+}$DM patients suggests that NOS2 and nitric oxide production may have protective and homeostatic effects. In this context, it was reported that nitric oxide can down-regulate immune responses, ${ }^{25,26}$ and NOS2 is especially required for muscle regeneration after damage. ${ }^{27}$ Interestingly, research on myocardial ischemia has highlighted the ability of cardiac myofibers to adapt to stress. Ischemic preconditioning is a biphasic phenomenon, ${ }^{28}$ which is protective against myocardial infarction and stunning. ${ }^{29}$ NOS2 and HSP70 are responsible for conferring protection during a subsequent ischemic challenge in vivo and in vitro. ${ }^{30-34}$ Thus, we hypothesize that NOS2 and HSP70 in MDA5 ${ }^{+}$patients may elicit reduced generation of reactive oxygen species (eg, in mitochondria) and enhanced cellular protection. Furthermore, we found that NOS2 colocalized with neural cell adhesion molecule in $\mathrm{MDA}^{+} \mathrm{DM}$ myofibers, which was not the case for classic DM patients. Neural cell adhesion molecule is mostly expressed by regenerating fibers in inflammatory myopathies. ${ }^{35}$ Thus, our results suggest that NOS2 expression is associated with regenerating effects.

To conclude, we show that muscle pathology in MDA5 ${ }^{+}$ DM does not merely represent an attenuated form of classic DM on the basis of the morphological picture, distribution, and phenotyping of immune cells and signs of vasculopathy as well IFN signature. Contrarily, we document a striking colocalization of NOS2 and HSP70 muscle fibers, which we interpret as protective because this association was shown to be involved in tissue homeostasis.

\section{Acknowledgments}

We thank the following members of the French Myositis Network: Arnaud Hot, Aurélie Grados, Nicolas Schleinitz, Laure Gallet, Nathalie Streichenberger, Philippe Petiot, Eric Hachulla, David Launay, Hervé Devilliers, Mohamed Hamidou, Divy Cornec, Boris Bienvenu, Vincent Langlois, Hervé Levesque, Aurélien Delluc, Laurent
Drouot, Jean-Luc Charuel, Fabienne Jouen, Norma Romero, Odile Dubourg, Sarah Leonard-Louis, Anthony Behin, Pascal Laforet, Tania Stojkovic, Bruno Eymard, Nathalie CostedoatChalumeau, Emmanuelle Campana-Salort, Anne Tournadre, Lucile Musset, Brigitte Bader-Meunier, Isabelle Kone-Paut, Jean Sibilia, Laurent Servais, Olivier Fain, Claire Larroche, Elizabeth Diot, Benjamin Terrier, Raphaël De Paz, Antoine Dossier, Dominique Menard, Chafika Morati, Marielle Roux, Xavier Ferrer, Jeremy Martinet, Sophie Besnard, Rémi Bellance, Patrice Cacoub, David Saadoun, Laurent Arnaud, Bernard Grosbois, Serge Herson, and Olivier Boyer.

Y.A. and W.S. conceived and designed the study, analyzed data, and wrote the manuscript; G.L., C.P., X.S.C., D.P., and E.G. performed histological analysis; A.R., B.H., M.H., N.L., A.M., T.M., and J.A. designed and coordinated the study and wrote the manuscript; H.-H.G. designed the study, assessed biopsy specimens, and wrote the manuscript; P.H. and N.Z. acquired and analyzed data; O.B. conceived, designed, and coordinated the study, and wrote the manuscript. All authors read and approved the final manuscript.

\section{Supplemental Data}

Supplemental material for this article can be found at http://dx.doi.org/10.1016/j.ajpath.2015.11.010.

\section{References}

1. Hoogendijk JE, Amato AA, Lecky BR, Choy EH, Lundberg IE, Rose MR, Vencovsky J, de Visser M, Hughes RA: 119th ENMC international workshop: trial design in adult idiopathic inflammatory myopathies, with the exception of inclusion body myositis, 10-12 October 2003, Naarden, The Netherlands. Neuromuscul Disord 2004, 14:337-345

2. De Visser M, Emslie-Smith AM, Engel AG: Early ultrastructural alterations in adult dermatomyositis: capillary abnormalities precede other structural changes in muscle. J Neurol Sci 1989, 94:181-192

3. Greenberg SA, Pinkus JL, Pinkus GS, Burleson T, Sanoudou D, Tawil R, Barohn RJ, Saperstein DS, Briemberg HR, Ericsson M, Park P, Amato AA: Interferon-alpha/beta-mediated innate immune mechanisms in dermatomyositis. Ann Neurol 2005, 57:664-678

4. Salajegheh M, Kong SW, Pinkus JL, Walsh RJ, Liao A, Nazareno R, Amato AA, Krastins B, Morehouse C, Higgs BW, Jallal B, Yao Y, Sarracino DA, Parker KC, Greenberg SA: Interferon-stimulated gene 15 (ISG15) conjugates proteins in dermatomyositis muscle with perifascicular atrophy. Ann Neurol 2010, 67:53-63

5. Gitiaux C, Kostallari E, Lafuste P, Authier FJ, Christov C, Gherardi RK: Whole microvascular unit deletions in dermatomyositis. Ann Rheum Dis 2013, 72:445-452

6. Sontheimer RD: Dermatomyositis: an overview of recent progress with emphasis on dermatologic aspects. Dermatol Clin 2002, 20:387-408

7. Hamaguchi Y, Kuwana M, Hoshino K, Hasegawa M, Kaji K, Matsushita T, Komura K, Nakamura M, Kodera M, Suga N, Higashi A, Ogusu K, Tsutsui K, Furusaki A, Tanabe H, Sasaoka S, Muro Y, Yoshikawa M, Ishiguro N, Ayano M, Muroi E, Fujikawa K, Umeda Y, Kawase M, Mabuchi E, Asano Y, Sodemoto K, Seishima M, Yamada H, Sato S, Takehara K, Fujimoto M: Clinical correlations with dermatomyositis-specific autoantibodies in adult Japanese patients with dermatomyositis: a multicenter cross-sectional study. Arch Dermatol 2011, 147:391-398 
8. Hall JC, Casciola-Rosen L, Samedy LA, Werner J, Owoyemi K, Danoff SK, Christopher-Stine L: Anti-MDA5-associated dermatomyositis: expanding the clinical spectrum. Arthritis Care Res Hoboken 2013, 65:1307-1315

9. Tansley SL, Betteridge ZE, Gunawardena H, Jacques TS, Owens CM, Pilkington C, Arnold K, Yasin S, Moraitis E, Wedderburn LR, McHugh NJ; UK Juvenile Dermatomyositis Research Group: AntiMDA5 autoantibodies in juvenile dermatomyositis identify a distinct clinical phenotype: a prospective cohort study. Arthritis Res Ther 2014, 16:R138

10. Saketkoo LA, Mittoo S, Huscher D, Khanna D, Dellaripa PF, Distler O, et al: Connective tissue disease related interstitial lung diseases and idiopathic pulmonary fibrosis: provisional core sets of domains and instruments for use in clinical trials. Thorax 2014, 69:428-436

11. Zerbe N, Hufnagl P, Schlüns K: Distributed computing in image analysis using open source frameworks and application to image sharpness assessment of histological whole slide images. Diagn Pathol 2011, 6:S16

12. Engel A, Franzini-Armstrong C: Myology, Basic and Clinical. Vol 1, ed 2. New York, NY: McGraw-Hill Medical Publishing, 1994

13. Preusse C, Goebel HH, Held J, Wengert O, Scheibe F, Irlbacher K, Koch A, Heppner FL, Stenzel W: Immune-mediated necrotizing myopathy is characterized by a specific Th1-M1 polarized immune profile. Am J Pathol 2012, 181:2161-2171

14. Rice GI, Forte G, Szynkiewicz M, Chase DS, Aeby A, AbdelHamid MS, et al: Assessment of interferon-related biomarkers in Aicardi-Goutières syndrome associated with mutations in: a casecontrol study. Lancet Neurol 2013, 12:1159-1169

15. Oestreich KJ, Weinmann AS: Transcriptional mechanisms that regulate T helper 1 cell differentiation. Curr Opin Immunol 2012, 24:191-195

16. Sica A, Mantovani A: Macrophage plasticity and polarization: in vivo veritas. J Clin Invest 2012, 122:787-795

17. Sato S, Hirakata M, Kuwana M, Suwa A, Inada S, Mimori T, Nishikawa T, Oddis CV, Ikeda Y: Autoantibodies to a 140-kd polypeptide, CADM-140, in Japanese patients with clinically amyopathic dermatomyositis. Arthritis Rheum 2005, 52:1571-1576

18. Cosnes A, Amaudric F, Gherardi R, Verroust J, Wechsler J, Revuz J, Roujeau JC: Dermatomyositis without muscle weakness: long-term follow-up of 12 patients without systemic corticosteroids. Arch Dermatol 1995, 131:1381-1385

19. Christov C, Chrétien F, Abou-Khalil R, Bassez G, Vallet G, Authier FJ, Bassaglia Y, Shinin V, Tajbakhsh S, Chazaud B, Gherardi RK: Muscle satellite cells and endothelial cells: close neighbors and privileged partners. Mol Biol Cell 2007, 18:1397-1409

20. Jerusalem F, Rakusa M, Engel AG, MacDonald RD: Morphometric analysis of skeletal muscle capillary ultrastructure in inflammatory myopathies. J Neurol Sci 1974, 23:391-402

21. Suárez-Calvet X, Gallardo E, Nogales-Gadea G, Querol L, Navas M, Díaz-Manera J, Rojas-Garcia R, Illa I: Altered RIG-I/DDX58-mediated innate immunity in dermatomyositis: RIG-I/DDX58 in dermatomyositis. J Pathol 2014, 233:258-268

22. Eloranta M-L, Barbasso Helmers S, Ulfgren A-K, Rönnblom L, Alm GV, Lundberg IE: A possible mechanism for endogenous activation of the type I interferon system in myositis patients with anti-Jo-1 or anti-Ro 52/anti-Ro 60 autoantibodies. Arthritis Rheum 2007, 56:3112-3124

23. Agarwal AK, Xing C, DeMartino GN, Mizrachi D, Hernandez MD, Sousa AB, Martínez de Villarreal L, dos Santos HG, Garg A: PSMB8 encoding the $\beta 5 \mathrm{i}$ proteasome subunit is mutated in joint contractures, muscle atrophy, microcytic anemia, and panniculitis-induced lipodystrophy syndrome. Am J Hum Genet 2010, 87:866-872

24. Crow YJ: Aicardi-Goutières syndrome. Handb Clin Neurol 2013, 113 : 1629-1635

25. Bogdan C: Nitric oxide and the immune response. Nat Immunol 2001, 2:907-916

26. Bronte V, Zanovello P: Regulation of immune responses by L-arginine metabolism. Nat Rev Immunol 2005, 5:641-654

27. Rigamonti E, Touvier T, Clementi E, Manfredi AA, Brunelli S, Rovere-Querini P: Requirement of inducible nitric oxide synthase for skeletal muscle regeneration after acute damage. J Immunol 2013, 190: 1767-1777

28. Marber MS, Latchman DS, Walker JM, Yellon DM: Cardiac stress protein elevation 24 hours after brief ischemia or heat stress is associated with resistance to myocardial infarction. Circulation 1993, 88: $1264-1272$

29. Bolli R: The early and late phases of preconditioning against myocardial stunning and the essential role of oxyradicals in the late phase: an overview. Basic Res Cardiol 1996, 91:57-63

30. Guo Y, Jones WK, Xuan YT, Tang XL, Bao W, Wu WJ, Han H, Laubach VE, Ping P, Yang Z, Qiu Y, Bolli R: The late phase of ischemic preconditioning is abrogated by targeted disruption of the inducible $\mathrm{NO}$ synthase gene. Proc Natl Acad Sci U S A 1999, 96:11507-11512

31. Plumier JC, Ross BM, Currie RW, Angelidis CE, Kazlaris H, Kollias G, Pagoulatos GN: Transgenic mice expressing the human heat shock protein 70 have improved post-ischemic myocardial recovery. J Clin Invest 1995, 95:1854-1860

32. Sur A, Kesaraju S, Prentice H, Ayyanathan K, Baronas-Lowell D, Zhu D, Hinton DR, Blanks J, Weissbach H: Pharmacological protection of retinal pigmented epithelial cells by sulindac involves PPAR- $\alpha$. Proc Natl Acad Sci U S A 2014, 111:16754-16759

33. Moench I, Prentice H, Rickaway Z, Weissbach H: Sulindac confers high level ischemic protection to the heart through late preconditioning mechanisms. Proc Natl Acad Sci U S A 2009, 106:19611-19616

34. Yin C, Salloum FN, Kukreja RC: A novel role of microRNA in late preconditioning: upregulation of endothelial nitric oxide synthase and heat shock protein 70. Circ Res 2009, 104:572-575

35. Winter A, Bornemann A: NCAM, vimentin and neonatal myosin heavy chain expression in human muscle diseases. Neuropathol Appl Neurobiol 1999, 25:417-424 